\title{
Phylloid Pattern of Hypomelanosis Closely Related to Chromosomal Abnormalities in the 13q Detected by SNP Array Analysis
}

\author{
F. Faletra ${ }^{a} \quad$ I. Berti ${ }^{a} \quad$ A. Tommasini ${ }^{a} \quad$ V. Pecile ${ }^{a} \quad$ L. Cleva ${ }^{a} \quad$ E. Alberini ${ }^{a}$ b $\quad$ I. Bruno \\ P. Gasparini ${ }^{a, b}$

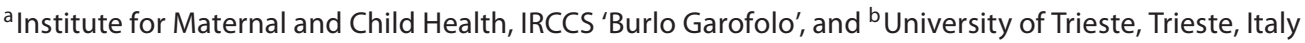

\author{
Key Words \\ Phylloid hypomelanosis - Mosaicism $\cdot 13 q \cdot$ \\ Deletion - SNP array analysis
}

\begin{abstract}
Phylloid hypomelanosis is a distinct type of pigmentary mosaicism characterized by congenital hypochromic macules resembling a floral ornament with various elements such as round or oval patches, asymmetrical macules similar to begonia leaves, or oblong lesions. It has been found to be predominantly associated with abnormalities in chromosome 13 and sometimes associated with different extracutaneous abnormalities. Here, we report 2 new cases of phylloid hypomelanosis due to mosaicism involving chromosome 13 . The first one is a mosaicism for a supernumerary marker belonging to chromosome 13 and the second one is the first report of phylloid hypomelanosis associated with a mosaic deletion of 13q. Because of the extremely low level of mosaicism in these 2 cases, SNP array analysis on skin fibroblasts was carried out, showing a 13q21.33-q34 duplication $(71,024,411-$ $115,103,529)$ and a $13 q 13.3-q 34(38,368,012-$ $115,103,529)$ deletion. Both cases underline on the one hand the strict connection between phylloid hypomelanosis and anomalies of chromosome 13 , and on the other
\end{abstract}

hand the relevance of the SNP array analysis on skin fibroblasts in the detection of lowlevel mosaicism.

Copyright $\odot 2012$ S. Karger AG, Basel

\section{Introduction}

Mosaicism refers to the occurrence in an individual of two or more cell populations that are karyotypically or genotypically different and yet are derived from a single zygote. An association between pigmentary anomalies and chromosomal mosaicism has been reported since the 1960 s $[1,2]$. Various mosaic pigment distributions are known, such as the lines of Blaschko, the checkerboard pattern, the phylloid pattern and the patchy pattern without midline separation [3]. In 1993, the phylloid pattern was delineated as a peculiar type of pigmentary mosaicism characterized by macules reminiscent of floral ornaments in the form of round or oval spots, patches resembling the asymmetric leaves of a begonia, and oblong lesions [4]. In contrast to the better known hypomelanosis of Ito (or 'mosaic hypomelanosis' as seen in hypomelanosis of Ito), which represents a cutaneous symptom of many different chromosomal abnormalities, phylloid hypomelanosis has been found to be predominantly associated with abnormalities in chromosome 13 [5]. Subsequently, the term phylloid hypomelanosis was suggested to define a condition consisting of achromic phylloid skin lesions in combination with extracutaneous anomalies such as mental retardation, agenesis of the corpus callosum, conductive hearing loss, coloboma, and various skeletal defects [6]. Here, we describe two new cases with phylloid hypomelanosis due to different chromosomal abnormalities. The first one showed mosaicism for a supernumerary marker belonging to the chromosome 13 and the second one showed mosaicism for a large deletion of the same chromosome. Both cases showed a very low level of mosaicism, and in the first one the marker was even too small to be recognized with a normal analysis of karyotype. An SNP array analysis performed on DNA from skin biopsy allowed us to make a correct diagnosis.

\section{Case Reports}

\section{Clinical Report 1}

The patient was an Italian boy born to nonconsanguineous mating. Due to advanced maternal age, amniocentesis was performed during the pregnancy; it showed

\section{KARGER}

E-Mail karger@karger.com

www.karger.com/drm
(C) 2012 S. Karger AG, Basel

1018-8665/12/2254-0294\$38.00/0
Flavio Faletra, MD

Medical Genetics, Department of Reproductive Sciences and Development Institute of Child Health, IRCCS 'Burlo Garofolo'

Via dell'Istria 65, IT-34137 Trieste (Italy)

E-Mail faletra@burlo.trieste.it 

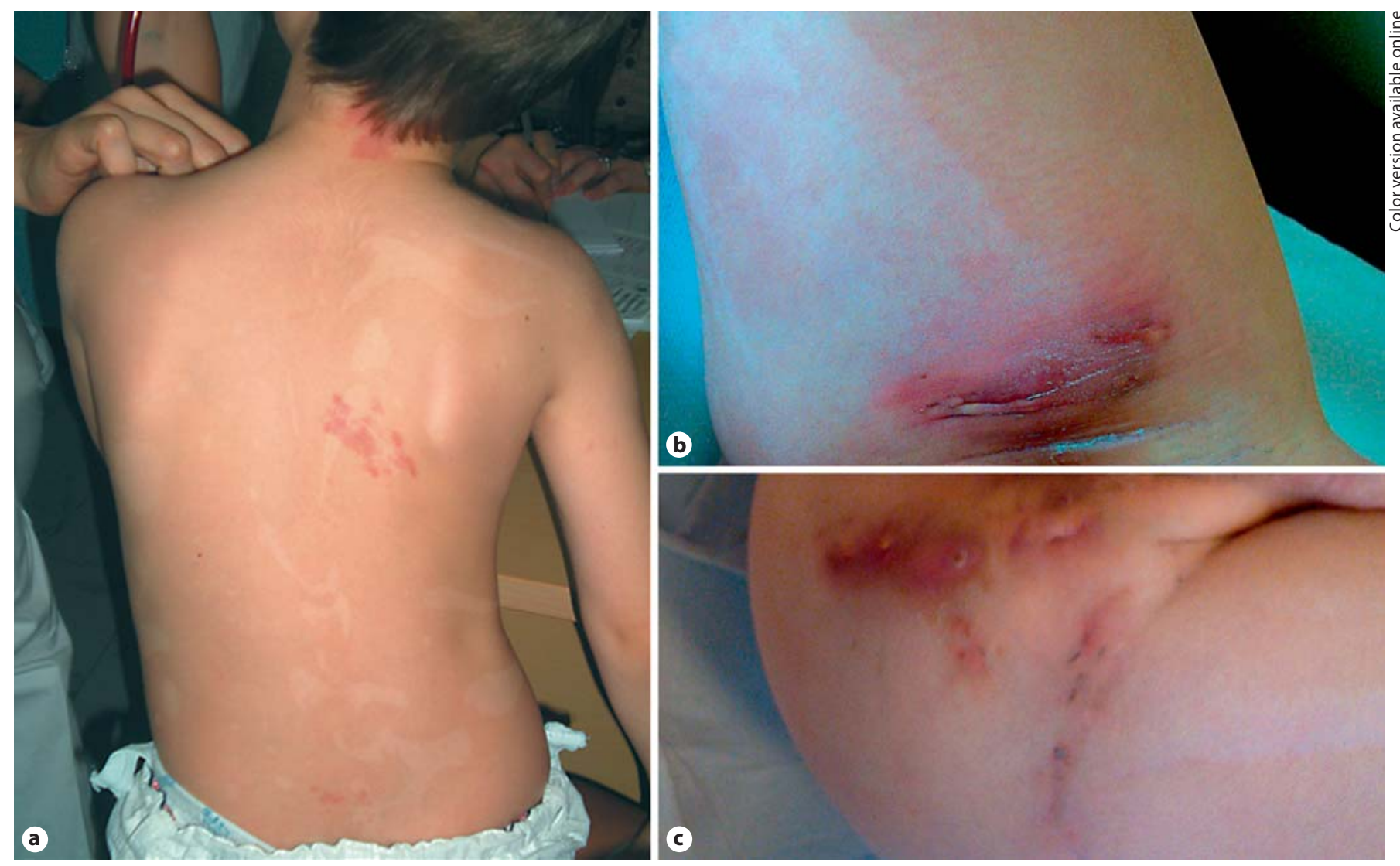

Fig. 1. a Asymptomatic and asymmetrical leaf-shaped hypomelanosis in a phylloid pattern, hypopigmented streaks and asymptomatic hypopigmented macules on the trunk and extremities. Note the telangiectatic macules on the trunk and the neck. $\mathbf{b}$, $\mathbf{c}$ Lesions reminiscent of hidradenitis suppurativa located in the hypomelanotic areas.

a karyotype $47, \mathrm{XY}+$ mar in 13 out of 64 metaphases from 3 different cell lines. The remaining 51 metaphases showed a normal karyotype, 46,XY. Morphology ultrasound evaluation revealed a hypoplastic stomach, while all the anthropometric measures were normal. The karyotype performed at birth on peripheral blood lymphocytes showed $2 \%$ of cells with the supernumerary marker. Because of the minimal size of the marker, it was not possible to detect the region or chromosome involved. At birth, length, weight and occipitofrontal circumference were all around the 50 th centile. At birth, the presence of diffuse hypomelanotic streaks, but no dysmorphic features except thick eyebrows, was noted. Vertebral schisis, a slight psychomotor delay and recurrent infections with abscesses in the hypomelanotic areas were referred. A brain MRI detected no malformations. Because of this medical history, he was re-

Phylloid Hypomelanosis and Mosaic 13q Abnormalities ferred to a medical genetics unit at the age of 3.5 years. A physical examination showed asymptomatic and asymmetrical leaf-shaped hypomelanosis in a phylloid pattern, hypopigmented streaks and asymptomatic hypopigmented macules on the trunk and extremities, and a medial telangiectatic nevus on the back (fig. 1a). Moreover, on the hypopigmented regions, several inflammatory lesions reminiscent of hidradenitis suppurativa were noted (fig. 1b, c). To detect the origin of the marker, an SNP array analysis on DNA from skin fibroblasts was carried out, showing a duplication of approximately $44 \mathrm{Mb}$ $(71,024,411-115,103,529)$ involving the region q21.33-q34 of the chromosome 13, and about $50 \%$ mosaicism in this tissue (fig. 2).

\section{Clinical Report 2}

The patient was the first-born to nonconsanguineous mating. During the preg- nancy, polyhydramnios was referred. The Apgar score was 9/10 at the first and 5th minute. The milestones were all delayed: head control was reached at 6 months, independent sitting at 14 months and walking at 26 months. He never developed speech and language. The phenotypic evaluation detected several dysmorphisms, including a triangular face, sparse eyebrows, upslanting palpebral fissures with ptosis in the right eye, and lowset and posteriorly rotated ears. At 3 years of age, all the anthropometric values were under the 3rd centile. Moreover, a phylloid pattern of multiple achromic macules on the trunk and limbs associated with a cherry telangiectatic macule on the scalp was noted. Additional features were cryptorchidism, hypoplasia of the 4 th and 5 th finger in the hands, and agenesis of the corpus callosum and an abnormal cortical gyration. Given the complexity of the clin- 


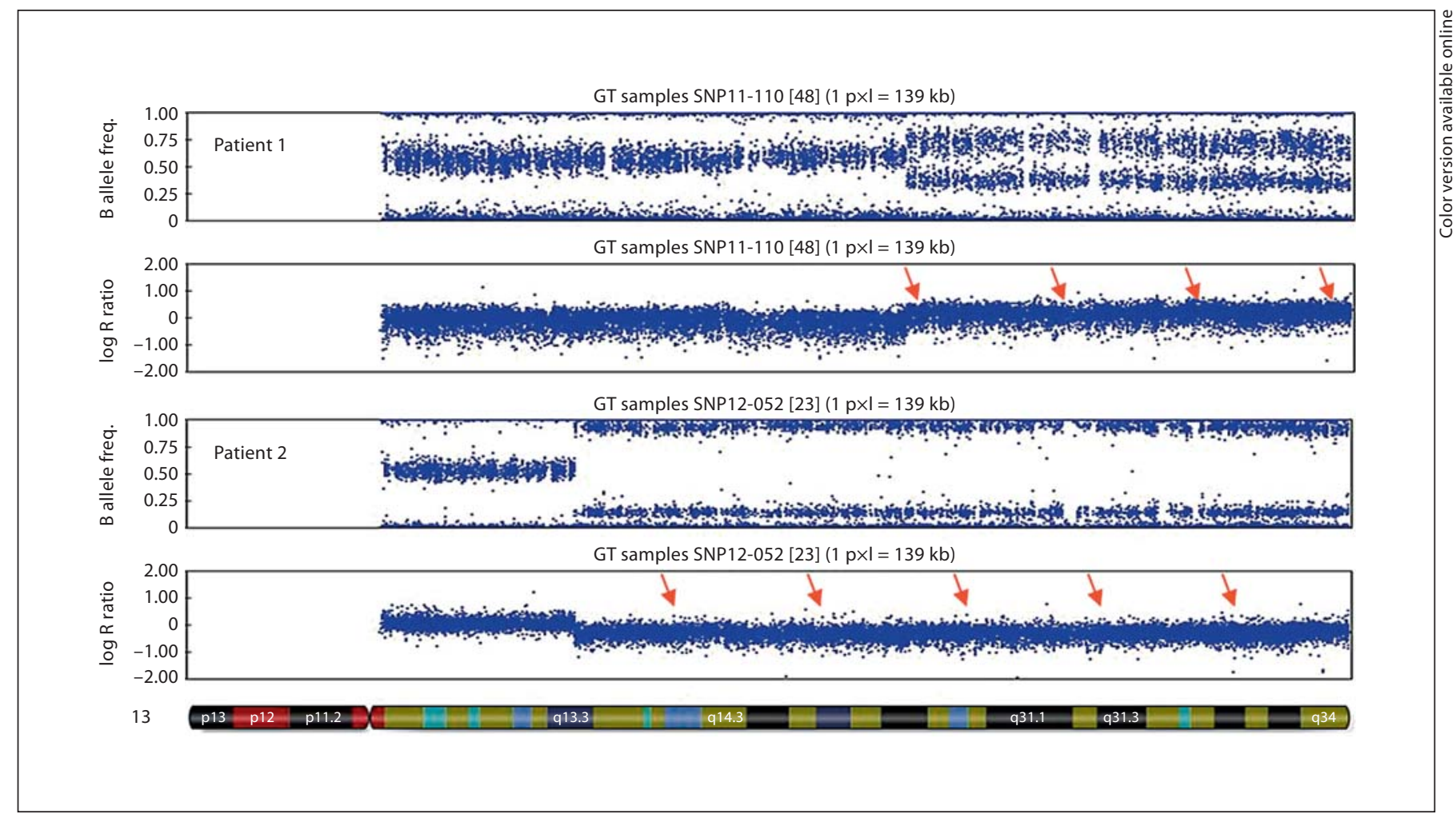

Fig. 2. SNP array analysis of chromosome 13. Arrows show the mosaic duplication in patient 1 and the mosaic deletion in patient 2.

ical picture, an SNP array analysis on DNA from lymphocytes was performed. The study demonstrated only a small inherited duplication of around $200 \mathrm{~Kb}(28,733,550$ $28,950,951)$ in the region p11.2 of the chromosome 16. Given the clinical severity and the multiple malformations on the one hand and the small duplication with its uncertain significance on the other, an SNP array analysis was performed on fibroblasts from a skin biopsy performed in the hypomelanotic areas. The SNP array analysis showed a high percentage of mosaicism $(80 \%)$ characterized by a deletion of around $76 \mathrm{Mb}(38,368,012-115,103,529)$ of the long arm of chromosome 13 (fig. 2).

\section{Materials and Methods}

Skin biopsy was performed at the level of the hypomelanotic macules. The karyotype analysis was performed on fibroblasts obtained from a culture of skin biopsy, according to standard procedures using QFQ banding techniques at 400 band resolution. SNP array analysis, from DNA isolated from peripheral blood lymphocytes and fibroblasts, was carried out using the Illumina HumanOmniExpress genotyping microarray according to the protocol. Data analysis was performed using the Illumina GenomeStudio v.2011.1 software program, and then we utilized the data with the program including Illumina cnvPartition (ver. 3.1.6). Informed consent was taken for each genetic analysis and for the publication of the pictures.

\section{Discussion}

Mosaic skin conditions do not always follow the lines of Blaschko. Happle [6] described four additional patterns of cutaneous mosaicism: block-like (type 2), phylloid (type 3), large patches without midline separation (type 4), and lateralization (type 5). The pattern of cutaneous mosaicism depends on the type of cell that is affected and its trajectory of migration and proliferation during embryogenesis. The phylloid pattern is composed of leaf-like, pearshaped, and oblong lesions in an arrange- ment reminiscent of a floral ornament. Although it is less common, the hyperpigmentation in a phylloid pattern has also been described and linked to mosaic tetrasomy 5 p, as well as chromosome 13 abnormalities [7]. In this case, the localization of phylloid macules is the same as in hypomelanosis. Moreover, it is possible to find in the same patient areas with phylloid hypochromic and hyperchromic patches.

Phylloid hypomelanosis refers to a distinct clinical entity with additional features that may include telangiectatic macules (fig. 1), hypertrichosis (e.g. thick eyebrows, long eyelashes, and a low frontal hairline), mental retardation, seizures, agenesis of the corpus callosum, conductive hearing loss, colobomas, and skeletal defects (especially of the digits) that are commonly observed in both mosaic and nonmosaic trisomy 13 [8]. Moreover, the median telangiectatic patches have been demonstrated to be more frequently associated with multiple congenital anomalies/ mental retardation [9].

These features as well as the presence of recurrent infections evolving in abscesses 
in the hypomelanotic areas are shared by our patient 1. Inflammatory lesions reminiscent of hidradenitis suppurativa have been previously described in 1 patient only [10]. It is still unclear if this condition is related to mosaic aberrations of the chromosome 13. The localization of the abscesses exclusively in the hypomelanotic areas prompted us to presume a connection between the mosaic duplication and the inflammatory lesions. Moreover, chronic cutaneous infections with abscesses affecting the parotid region, axillae, groin, and abdominal wall, occasionally reported in Patau syndrome, have been related to defects in neutrophil shape and function [11].

To our knowledge, this is the first report of phylloid hypomelanosis related to a 13q mosaic deletion. Although the skin condition can be explained by the mosaic copy number variation, the general clinical picture may also be related to the 16 p11.2 duplication, responsible for developmental delay and obesity [12].

Moreover, except for a case of tetrasomy $13 q$ mosaicism [13], this is the first report of phylloid hypomelanosis analyzed with microarray, a technique able to define accurately the breakpoints and the genes belonging to the microduplication/deletion. Several candidate pigmentary genes have been identified in the genome. Three of them are located within the minimal area detected in patients with phylloid hypomelanosis. EDNRB (endothelin receptor type B) maps to $13 \mathrm{q} 22$ and functions as a growth factor receptor responsible for melanoblast migration [14]. It is also involved in Waardenburg syndrome, characterized by dyschromic patches in the skin, heterochromia iridis and white forelock. Another gene is the DCT (dopachrome tautomerase; on 13q32), which functions as a melanosomal enzyme. The homologous gene mutated in mice has been demonstrated to be responsible for the phenotype of 2 slaty mice [15]. The last gene is the EFNB2 (ephrin-B2; on 13q33), which is a growth factor also responsible for melanoblast migration [16].

A possible explanation for this specific skin phenotype is that mosaic expression of these genes may cause an impaired melanoblast migration and melanocyte formation leading to the depigmentation.

Although several authors assert the strict connection between the abnormalities of chromosome 13 and the hypo/hypermelanotic changes in the skin of patients, the underlying pathogenetic mechanism is still unclear. Moreover, the new correlation between the mosaic deletion of chromosome 13 and the phylloid hypomelanosis leads one to hypothesize complex interactions between genes rather than simply a dose effect.

\section{Conclusions}

Our 2 cases provide additional evidence that phylloid hypomelanosis comprises multiple birth defects and a distinct entity. When a phylloid pattern is present, we should be able to interpret these signs and foretell the presence of a mosaic deletion/ duplication of chromosome 13. In this field a SNP array analysis on DNA from skin fibroblasts can be very useful to inform about the size and percentage of mosaicism. Further functional studies are required to elucidate the pathogenesis of phylloid hypomelanosis and the relationship with the hidradenitis suppurativa-like lesions.

\section{Acknowledgements}

The authors thank the patients and their parents who participated in the study for their continuous support. Moreover, the authors deeply appreciate Mario Cutrone and Carmelo Schepis for their thorough revision of the dermatological features of our patients.

\section{Disclosure Statement}

The authors declare no conflicts of interest.

\section{References}

$\checkmark 1$ Ferrier P, Stadler G, Bamatter F, Ferrier S, Buehler E, Klein D, Congenital asymmetry associated with diploid-triploid mosaicism and large satellites. Lancet 1964;1:80-82.

$\checkmark 2$ Zuelzer WW, Beattie KM, Reisman LE: Generalized unbalanced mosaicism attributable to dispermy and probable fertilization of a polar body. Hum Genet 1964;16:38-51.

$\checkmark 3$ Happle R: Mosaicism in human skin: understanding the patterns and mechanisms. Arch Dermatol 1993;129:1460-1470.

$\checkmark 4$ Happle R: Phylloid hypomelanosis and mosaic trisomy 13: a new etiologically defined neurocutaneous syndrome (in German). Hautarzt 2001;52:3-5.

$\checkmark 5$ Happle R: Phylloid hypomelanosis is closely related to mosaic trisomy 13. Eur J Dermatol 2000;10:511-512.

6 Happle R: Dohi Memorial Lecture. New aspects of cutaneous mosaicism. J Dermatol 2002;29:681-692.

$>7$ Oiso N, Tsuruta D, Imanishi H, Sayasa H, Narita T, Kobayashi H, Ikegami H, Kawada A: Phylloid hypermelanosis and melanocytic nevi with aggregated and disfigured melanosomes: causal relationship between phylloid pigment distribution and chromosome $13 \mathrm{ab}-$ normalities. Dermatology 2010;220:169-172.

8 Delatycki M, Gardner RJ: Three cases of trisomy 13 mosaicism and a review of the literature. Clin Genet 1997;51:403-407.

$\checkmark$ Schepis C, Greco D, Failla P, Siragusa M, Romano C, Scaffidi M, Sindoni A: Medial telangiectatic sacral nevi and MCA/MR syndromes. Pediatr Dermatol 2003;20:370-371.

10 González-Enseñat MA, Vicente A, Poo P, Catalá V, Mar Pérez-Iribarne M, Fuster C, Geán E, Happle R: Phylloid hypomelanosis and mosaic partial trisomy 13: two cases that provide further evidence of a distinct clinicogenetic entity. Arch Dermatol 2009;145: 576-578.

11 Huehns ER, Lutzner M, Hecht F: Nuclear abnormalities of the neutrophils in $\mathrm{d} 1$ (13-15)-trisomy syndrome. Lancet 1964;1: 589-590.

12 Bachmann-Gagescu R, Mefford HC, Cowan C, Glew GM, Hing AV, Wallace S, Bader PI,
Hamati A, Reitnauer PJ, Smith R, Stockton DW, Muhle H, Helbig I, Eichler EE, Ballif BC, Rosenfeld J, Tsuchiya KD: Recurrent $200-\mathrm{kb}$ deletions of $16 \mathrm{p} 11.2$ that include the SH2B1 gene are associated with developmental delay and obesity. Genet Med 2010; 12:641-647.

13 Dhar SU, Robbins-Furman P, Levy ML, Patel A, Scaglia F: Tetrasomy 13q mosaicism associated with phylloid hypomelanosis and precocious puberty. Am J Med Genet A 2009; 149A:993-996.

14 Sturm RA, Box NF, Ramsay M: Human pigmentation genetics: the difference is only skin deep. Bioessays 1998;20:712-721.

15 Budd PS, Jackson IJ: Structure of the mouse tyrosinase-related protein-2/dopachrome tautomerase (Tyrp2/Dct) gene and sequence of two novel slaty alleles. Genomics 1995;29: 35-43.

-16 Santiago A, Erickson CA: Ephrin-B ligands play a dual role in the control of neural crest cell migration. Development 2002;129:36213632 . 